\title{
Description of rotational molecular spectra by means of an approach based on rational mechanics
}

\author{
Elena A. Ivanova*, Anton M. Krivtsov, and Pavel A. Zhilin $\dagger$ \\ Department of Theoretical Mechanics, Faculty of Physics and Mechanics, St. Petersburg State Polytechnical University, \\ Polytechnicheskaya Street 29, 195251 St. Petersburg, Russia
}

Received 13 September 2006, revised 27 October 2006, accepted 12 December 2006

Published online 8 February 2007

Key words molecular spectra, diatomic, rigid body, continuum mechanics, oscillations

In majority of publications the spectrum of diatomic molecules in far infrared spectral area is described as a purely quantum phenomenon, and the spectrum in near infrared spectral area is described by means of using a semi-classical approach. In our work an attempt to describe the both spectra from mechanical point of view is presented. The approach is based on accounting of the inertia properties of an interatomic bond. In such approach a potential of interaction between atoms in a molecule is defined by a set of partial differential equations. Good agreement with known experimental data in far infrared spectral area and a qualitative explanation of a thin structure of a resonant peak in the near infrared spectral area is obtained.

(c) 2007 WILEY-VCH Verlag GmbH \& Co. KGaA, Weinheim

\section{Introduction}

Numerous experiments show that diatomic molecules have spectra in infrared area. In far infrared spectral area their spectra consist of a series of approximately equidistant lines [1-3]. Usually, the spectrum is considered as a rotational one due to the quantum mechanical model explaining the presence of this spectrum. In the near infrared spectral area the spectra consist, as a rule, of one, very intensive, line and of weak lines at double and triple frequencies [1-3]. This spectrum is usually referred to a vibrational one. The presence of this spectrum can be explained by oscillations of a molecule, which behaves like a harmonic oscillator. An intensive line in this case is associated with a resonant peak of an amplitude frequency characteristics of the oscillator. More detailed consideration of the resonant peak in the near infrared area shows that it has so-called thin structure, e.g. it consists of a set of close-located resonant peaks [1-3]. This thin structure is usually referred as vibrational-rotational.

Let us emphasize, that direct measurement allows to obtain the molecular spectra only. A correspondence between motion of molecule and this or that spectrum can be obtained only in an indirect way, by means a theoretical model allowing to obtain a satisfactory description of the spectra. Thus, the known eigenforms of molecular oscillations are based mainly on theoretical considerations, but not on experimental measurements.

In many cases, atomic systems can be adequately described as systems of rigid bodies (one-spin particles) interacting via forces and moments $[4,5]$. The character of interaction between one-spin particles is not precisely known. However, for a great number of molecules the oscillation spectrum is well-known. Obviously, the spectrum is tidily connected with the character of interaction between one-spin particles. The existence of an infinite spectrum shows that molecules can not be considered as systems with finite number of degrees of freedom. For example, a diatomic molecule can not be a system with 12 degrees of freedom only, as in the case with a system of two rigid bodies. This seems like a contradiction, since it is widely accepted that molecules are systems of atoms, i.e. systems with finite number of degrees of freedom. As a rule, the contradiction is used as an argument that such systems can be described only by means of a using quantummechanical approach. Indeed, in the frames of quantum mechanics, the infinite spectrum can be described as a consequence of rotational energy quantization. In the work, we show that the contradiction can be eliminated within the framework of classical mechanics, assuming that interaction between atoms in a molecule is not instantaneous, i.e. it is not described by a potential, which depends only on position and orientation of the atoms in a molecule. Similarly to electrodynamics, where potentials of interaction between charges can be described by solutions of partial differential equations, we assume that interaction potentials in molecules, as well, are determined by some partial differential equations.

\footnotetext{
* Corresponding author, E-mail: ivanova @ ei5063.spb.edu, Phone: +78123232762, Fax: +78123214771
} 
Let us name a molecule as $A$ body and the atoms in the molecule as bodies $A_{1}$ and $A_{2}$, respectively. Let us assume, that the bodies $A_{1}$ and $A_{2}$ are in a medium, which determines interaction between them. The bodies produce disturbances in the medium, which is of significance at the distances comparable with sizes of the atoms, but can be neglected at large distances. Let us name the medium under disturbance in the direct vicinity of the bodies $A_{1}$ and $A_{2}$ as the body $A_{3}$. We accept that the body $A$ contains also the body $A_{3}$. What kind of physical object is modelled by the body $A_{3}$ ? To answer the question, let us consider the following two models.

The first model. Assume that the bodies $A_{1}$ and $A_{2}$ includes atoms and electrons not participating in the valent bonding. The hybridized electron clouds forming the bond in a diatomic molecule we name as the body $A_{3}$. Such models are considered in quantum chemistry. An significant difference of this model from one used in quantum chemistry, is that the electron clouds are believed to be a continuous medium, which is described by partial differential equations.

The second model. Assume that the bodies $A_{1}$ and $A_{2}$ are the atoms, and the body $A_{3}$ is a part of a disturbed electromagnetic field, which lies in direct vicinity of the atoms. The disturbance in the electromagnetic field appears due to the fact that the atoms contain positive and negative charged particles. The model lies not so close to the traditional one, but it is in a good agreement with the criterion that an electromagnetic field and positive and negative charges interact with each other.

It is difficult to prefer this or that model, because the modern measuring equipment can give no possibility to discover internal structure of a molecule. The most reliable data, which can be obtained from experiment, are the molecular spectra. Therefore, any molecular model describing its spectra has the right to exist. For the purposes of particular concern of our work the physical interpretation of the molecular model is of no importance. The following is important. For the bodies $A_{1}$ and $A_{2}$, it is permissible to use models of one-spin particles (solid bodies), for the body $A_{3}$ it is important to use a continuous model.

For simplicity of the further work let us use the following three assumptions regarding the continuum model of the body $\left.A_{3}: 1\right)$ The considered medium is concentrated near the segment connecting atoms of the diatomic molecule, therefore for its modelling it is admissible to use an one-dimensional continuum. 2) The one-dimensional continuum consists from one-spin particles (rigid bodies) possessing six degrees of freedom and interacting via forces and moments. 3) The forces and moments depend only on position of these particles and do not depend on their velocities - in other words a model of elastic continuum is considered. Thus, the body $A$, modelling a molecule, includes two one-spin particles (the bodies $A_{1}$ and $A_{2}$ ) and one-dimensional elastic continuum (the body $A_{3}$ ). It is supposed, that the bodies $A_{1}$ and $A_{2}$ cannot interact directly, they interact with the body $A_{3}$ by means of which the interaction of these bodies is performed.

In the presented work the direct tensor calculus is used for description of the dynamics of one-spin particles (rigid bodies). In details description of this technique can be found in [6,7].

\section{One-spin particle model}

Position in space of an one-spin particle is determined by a vector of position $\boldsymbol{R}(t)$ and a tensor of rotation $\boldsymbol{P}(t)$. The tensor of rotation is a properly orthogonal tensor, which represents the solution of equations

$$
\boldsymbol{P} \cdot \boldsymbol{P}^{T}=\boldsymbol{P}^{T} \cdot \boldsymbol{P}=\boldsymbol{E}, \quad \operatorname{det} \boldsymbol{P}=1 ;
$$

where $\boldsymbol{E}$ is the unit tensor. Translational and angular velocities of an one-spin particle are calculated by formulas:

$$
\boldsymbol{v}(t)=\dot{\boldsymbol{R}}(t), \quad \boldsymbol{\omega}(t)=-\frac{1}{2}\left[\dot{\boldsymbol{P}}(t) \cdot \boldsymbol{P}^{T}(t)\right]_{\times},
$$

where $\boldsymbol{A}_{\times}$is the vector invariant of tensor $\boldsymbol{A}$. Kinetic energy $K^{*}$ of an one-spin particle by definition is the quadratic form of its velocities $[8,9]$, namely,

$$
K^{*}=m K, \quad K=\frac{1}{2} \boldsymbol{v} \cdot \boldsymbol{v}+\boldsymbol{v} \cdot \boldsymbol{\Theta}_{1} \cdot \boldsymbol{\omega}+\frac{1}{2} \boldsymbol{\omega} \cdot \boldsymbol{\Theta}_{2} \cdot \boldsymbol{\omega},
$$

where $m$ is the mass of an one-spin particle; $K$ is the mass density of kinetic energy; $\boldsymbol{\Theta}_{1}$ and $\boldsymbol{\Theta}_{2}$ are the mass densities of inertia tensors. Momentum $\boldsymbol{K}_{1}^{*}$ and angular momentum $\boldsymbol{K}_{2}^{*}$ of an one-spin particle are defined by formulas [8,9]:

$$
\boldsymbol{K}_{1}^{*}=m \boldsymbol{K}_{1}, \quad \boldsymbol{K}_{1}=\frac{\partial K}{\partial \boldsymbol{v}} ; \quad \boldsymbol{K}_{2}^{*}=m \boldsymbol{K}_{2}, \quad \boldsymbol{K}_{2}=\boldsymbol{R} \times \frac{\partial K}{\partial \boldsymbol{v}}+\frac{\partial K}{\partial \boldsymbol{\omega}} .
$$




\section{Fundamental laws of mechanics}

Definition. Let us consider a closed surface $S_{A}$. The set of one-spin particles $A_{i}$, being inside of surface $S_{A}$ we name as body $A$. Let us name the set of one-spin particles, being outside of surface $S_{A}$, as an environment of body $A$ and denote it as $A^{e}$. For simplicity, let us assume that no particles are present on surface $S_{A}$.

Kinetic energy, momentum, and angular momentum satisfy additivity axioms. According to the axioms for body $A$ consisting of a discrete set of bodies $A_{i}$ the following formulas are valid:

$$
K^{*}(A)=\sum_{i} K^{*}\left(A_{i}\right), \quad \boldsymbol{K}_{1}^{*}(A)=\sum_{i} \boldsymbol{K}_{1}^{*}\left(A_{i}\right), \quad \boldsymbol{K}_{2}^{*}(A)=\sum_{i} \boldsymbol{K}_{2}^{*}\left(A_{i}\right) .
$$

When the mass in body $A$ is continuously distributed, the sums in formulas (5) are substituted by integrals.

Let us formulate the fundamental laws of mechanics for the bodies, whose mass and energy of non-mechanical nature are not exchanged.

The first law of dynamics by Euler. A rate in the momentum change of body $A$ is equal to the force $\boldsymbol{F}\left(A, A^{e}\right)$ acting on the body $A$ from its environment, namely:

$$
\dot{\boldsymbol{K}}_{1}^{*}(A)=\boldsymbol{F}\left(A, A^{e}\right)
$$

The second law of dynamics by Euler. A rate in the angular momentum change of body $A$ is equal to the moment $M\left(A, A^{e}\right)$ acting on body $A$ from its environment:

$$
\dot{\boldsymbol{K}}_{2}^{*}(A)=\boldsymbol{M}\left(A, A^{e}\right) .
$$

Here, the angular momentum $\boldsymbol{K}_{2}^{*}(A)$ and the the external moment $\boldsymbol{M}\left(A, A^{e}\right)$ are calculated relative to the same reference points.

The third fundamental law of mechanics (the energy balance equation). A rate in in the total energy change of body $A$ is equal to the external force and moment power, namely:

$$
\dot{E}^{*}(A)=N\left(A, A^{e}\right), \quad E^{*}(A)=K^{*}(A)+U^{*}(A) .
$$

Here, $E^{*}(A)$ is the total energy of body $A ; U^{*}(A)$ is the internal energy of body $A ; N\left(A, A^{e}\right)$ is the the external force and moment power.

\section{Model of one-dimensional elastic continuum consisting of one-spin particles}

Let us consider an one-dimensional continuum model where each point represents an one-spin particle. In the reference configaration, all particles of the continuum are placed on a segment of line $0 \leq s \leq l$, i.e. their positions are determined by radius-vector $\boldsymbol{r}(s)=s \boldsymbol{\tau}$, where $\boldsymbol{\tau}$ is the unit vector. Rotation tensor of the particles in a reference configuration is believed to be unit. Position of particles in continuum for the actual configuration is set by position vector $\boldsymbol{R}(s, t)$ and rotation tensor $\boldsymbol{P}(s, t)$. The translational and angular velocities of particles in continuum are calculated by formulas (2). Now, we consider the dynamic structures of an one-dimensional continuum of one-spin particles. Kinetic energy, momentum, and angular momentum of a part of one-dimensional continuum located between cross-sections $s_{1}$ and $s_{2}$ are expressed via integrals:

$$
K^{*}=\int_{s_{1}}^{s_{2}} \rho K d s, \quad \boldsymbol{K}_{1}^{*}=\int_{s_{1}}^{s_{2}} \rho \boldsymbol{K}_{1} d s, \quad \boldsymbol{K}_{2}^{*}=\int_{s_{1}}^{s_{2}} \rho \boldsymbol{K}_{2} d s,
$$

where $\rho$ is the linear density; $K$ is the mass density of kinetic energy evaluated by formula (3); $\boldsymbol{K}_{1}, \boldsymbol{K}_{2}$ are the mass densities of momentum and angular momentum, respectively determined by formulas (4).

Let us formulate the fundamental laws of mechanics. An integral form of the first law of dynamics by Euler is

$$
\int_{s_{1}}^{s_{2}} \rho \dot{\boldsymbol{K}}_{1} d s=\int_{s_{1}}^{s_{2}} \rho \boldsymbol{F} d s+\boldsymbol{N}_{(\tau)}\left(s_{2}, t\right)+\boldsymbol{N}_{(-\tau)}\left(s_{1}, t\right),
$$

where $\boldsymbol{F}$ is the mass density of force acting from an environment, $\boldsymbol{N}_{(\tau)}\left(s_{2}, t\right)$ simulates the force acting from a part of the continuum on the right side of point $s_{2} ; \boldsymbol{N}_{(-\tau)}\left(s_{1}, t\right)$ simulates the force acting from a part of the continuum on the left side of point $s_{1}$. 
In formulation of the second law of dynamics by Euler, it is necessary to choose the reference point and the point of reduction. As for the reference point, we choose the reference frame origin, and as for the point of reduction let us choose the point $\boldsymbol{R}(s, t)$. An integral form of the second law of dynamics by Euler is

$$
\begin{aligned}
\int_{s_{1}}^{s_{2}} \rho \dot{\boldsymbol{K}}_{2} d s= & \int_{s_{1}}^{s_{2}} \rho[\boldsymbol{R} \times \boldsymbol{F}+\boldsymbol{L}] d s \\
& +\boldsymbol{R}\left(s_{2}, t\right) \times \boldsymbol{N}_{(\tau)}\left(s_{2}, t\right)+\boldsymbol{R}\left(s_{1}, t\right) \times \boldsymbol{N}_{(-\tau)}\left(s_{1}, t\right)+\boldsymbol{M}_{(\tau)}\left(s_{2}, t\right)+\boldsymbol{M}_{(-\tau)}\left(s_{1}, t\right),
\end{aligned}
$$

where $\boldsymbol{L}$ is the mass density of moment acting from an environment; $\boldsymbol{M}_{(\tau)}\left(s_{2}, t\right)$ simulates the moment acting from a part of the continuum on the right side of point $s_{2} ; M_{(-\tau)}\left(s_{1}, t\right)$ simulates the moment acting from a part of the continuum on the left side of point $s_{1}$.

Applying Eqs. (10), (11) to infinitesimal elements of the continuum, it is easy to prove a analogues of Newton's third law

$$
\boldsymbol{N}(s, t) \equiv \boldsymbol{N}_{(\tau)}(s, t)=-\boldsymbol{N}_{(-\tau)}(s, t), \quad \boldsymbol{M}(s, t) \equiv \boldsymbol{M}_{(\tau)}(s, t)=-\boldsymbol{M}_{(-\tau)}(s, t),
$$

which allows to rewrite Eqs. (10), (11) in the form

$$
\begin{aligned}
& \int_{s_{1}}^{s_{2}} \rho\left(\boldsymbol{v}+\boldsymbol{\Theta}_{1} \cdot \boldsymbol{\omega}\right)^{\cdot} d s=\int_{s_{1}}^{s_{2}}\left[\boldsymbol{N}^{\prime}+\rho \boldsymbol{F}\right] d s, \\
& \int_{s_{1}}^{s_{2}}\left[\rho \boldsymbol{v} \times \boldsymbol{\Theta}_{1} \cdot \boldsymbol{\omega}+\rho\left(\boldsymbol{v} \cdot \boldsymbol{\Theta}_{1}+\boldsymbol{\Theta}_{2} \cdot \boldsymbol{\omega}\right)^{\cdot}\right] d s=\int_{s_{1}}^{s_{2}}\left[\boldsymbol{M}^{\prime}+\boldsymbol{R}^{\prime} \times \boldsymbol{N}+\rho \boldsymbol{L}\right] d s .
\end{aligned}
$$

Using arbitrariness in our choice of cross-sections $s_{1}$ and $s_{2}$, we obtain from the above equations a local form of the laws of dynamics, namely:

$$
\begin{aligned}
& \rho\left(\boldsymbol{v}+\boldsymbol{\Theta}_{1} \cdot \boldsymbol{\omega}\right)^{\cdot}=\boldsymbol{N}^{\prime}+\rho \boldsymbol{F}, \\
& \rho \boldsymbol{v} \times \boldsymbol{\Theta}_{1} \cdot \boldsymbol{\omega}+\rho\left(\boldsymbol{v} \cdot \boldsymbol{\Theta}_{1}+\boldsymbol{\Theta}_{2} \cdot \boldsymbol{\omega}\right)^{\cdot}=\boldsymbol{M}^{\prime}+\boldsymbol{R}^{\prime} \times \boldsymbol{N}+\rho \boldsymbol{L} .
\end{aligned}
$$

The third fundamental law of mechanics (the energy balance equation) is of the form:

$$
\begin{aligned}
\int_{s_{1}}^{s_{2}} \rho(\dot{K}+\dot{U}) d s= & \int_{s_{1}}^{s_{2}} \rho[\boldsymbol{F} \cdot \boldsymbol{v}+\boldsymbol{L} \cdot \boldsymbol{\omega}] d s \\
& +\boldsymbol{N}_{(\tau)}\left(s_{2}, t\right) \cdot \boldsymbol{v}\left(s_{2}, t\right)+\boldsymbol{M}_{(\tau)}\left(s_{2}, t\right) \cdot \boldsymbol{\omega}\left(s_{2}, t\right)+\boldsymbol{N}_{(-\tau)}\left(s_{1}, t\right) \cdot \boldsymbol{v}\left(s_{1}, t\right) \\
& +\boldsymbol{M}_{(-\tau)}\left(s_{1}, t\right) \cdot \boldsymbol{\omega}\left(s_{1}, t\right),
\end{aligned}
$$

where $U$ is the mass density of internal energy. Using Eqs. (12), (15), (16), a local form of the energy balance equation can be obtained

$$
\rho \dot{U}=\boldsymbol{N} \cdot\left(\boldsymbol{v}^{\prime}+\boldsymbol{R}^{\prime} \times \boldsymbol{\omega}\right)+\boldsymbol{M} \cdot \boldsymbol{\omega}^{\prime}
$$

Let us introduce the strain vectors $\varepsilon, \boldsymbol{\Phi}$ and the energy vectors of strain $\varepsilon_{\times}, \boldsymbol{\Phi}_{\times}$

$$
\varepsilon=\boldsymbol{R}^{\prime}-\boldsymbol{P} \cdot \boldsymbol{\tau}, \quad \varepsilon_{\times}=\boldsymbol{P}^{T} \cdot \varepsilon, \quad \boldsymbol{P}^{\prime}=\boldsymbol{\Phi} \times \boldsymbol{P}, \quad \boldsymbol{\Phi}_{\times}=\boldsymbol{P}^{T} \cdot \boldsymbol{\Phi} .
$$

With the use of Eqs. (19) the energy balance equation (18) takes the form:

$$
\rho \dot{U}=\boldsymbol{N} \cdot \boldsymbol{P} \cdot \dot{\varepsilon}_{\times}+\boldsymbol{M} \cdot \boldsymbol{P} \cdot \dot{\boldsymbol{\Phi}}_{\times} \cdot
$$

Note, that all equations obtained above can be applied for any medium, which is not necessary elastic. An assumption of elasticity for the continuum considered is equivalent to the following statement: the density of internal energy, the vector of force, and the vector of moment depend on a configuration of the system at the present moment of time and do not depend on the time history of deformation. In other words, the density of internal energy, the vector of force, and the vector of moment depend only on vectors of strain and do not depend on velocities. By assumption of elasticity for the considered continuum together with equation of energy balance (20) allow the Cauchy-Green's relations can be derived:

$$
\boldsymbol{N}=\frac{\partial \rho U}{\partial \varepsilon_{\times}} \cdot \boldsymbol{P}^{T}, \quad \boldsymbol{M}=\frac{\partial \rho U}{\partial \boldsymbol{\Phi}_{\times}} \cdot \boldsymbol{P}^{T}
$$


To close the set of Eqs. (15), (19), (21), it is enough to set the density of internal energy of the medium as a strain vectors function. The simplest form of the internal energy is the quadratic form of the strain vectors, namely:

$$
\rho U\left(\varepsilon_{\times}, \boldsymbol{\Phi}_{\times}\right)=\frac{1}{2} \varepsilon_{\times} \cdot \boldsymbol{A} \cdot \varepsilon_{\times}+\varepsilon_{\times} \cdot \boldsymbol{B} \cdot \boldsymbol{\Phi}_{\times}+\frac{1}{2} \boldsymbol{\Phi}_{\times} \cdot \boldsymbol{C} \cdot \boldsymbol{\Phi}_{\times},
$$

where $\boldsymbol{A}, \boldsymbol{B}, \boldsymbol{C}$ are the stiffness tensors i.e. the quantities, which depend on the properties of the medium and do not depend on strains. With the use of (22) the Eqs. (21) can be rewritten in the form

$$
\boldsymbol{N}=\left(\boldsymbol{A} \cdot \boldsymbol{\varepsilon}_{\times}+\boldsymbol{B} \cdot \boldsymbol{\Phi}_{\times}\right) \cdot \boldsymbol{P}^{T}, \quad \boldsymbol{M}=\left(\varepsilon_{\times} \cdot \boldsymbol{B}+\boldsymbol{C} \cdot \boldsymbol{\Phi}_{\times}\right) \cdot \boldsymbol{P}^{T} .
$$

Thus, Eqs. (15), (16), (19), and (23) describe the dynamics of one-dimensional elastic continuum of one-spin particles. Note, that these equations, from mathematical point of view, fully coincide with equations of the general theory of rods, that includes all forms of deformations [9].

\section{Continuous model of diatomic molecule}

Now, we consider model of diatomic molecule, including two one-spin particles (bodies $A_{1}$ and $A_{2}$ ), modelling atoms, and an one-dimensional elastic continuum of one-spin particles (body $A_{3}$ ), simulating the medium by means of which the atoms interact with each other. Dynamics of body $A_{3}$ is described by Eqs. (15), (16), (19), (23). The equations of dynamics for bodies $A_{1}$ and $A_{2}$ have the form of:

$$
\dot{\boldsymbol{K}}_{1}^{*}\left(A_{i}\right)=\boldsymbol{F}\left(A_{i}, A_{3}\right), \quad \dot{\boldsymbol{K}}_{2}^{*}\left(A_{i}\right)=\boldsymbol{M}\left(A_{i}, A_{3}\right), \quad i=1,2 .
$$

Assume, that the particles of the continuum, which lie in vicinity of bodies $A_{1}$ and $A_{2}$, are so strongly attracted to the bodies, that the following conditions are to be fulfilled:

$$
\boldsymbol{R}_{1}(t)=\left.\boldsymbol{R}(s, t)\right|_{s=0}, \quad \boldsymbol{P}_{1}(t)=\left.\boldsymbol{P}(s, t)\right|_{s=0}, \quad \boldsymbol{R}_{2}(t)=\left.\boldsymbol{R}(s, t)\right|_{s=l}, \quad \boldsymbol{P}_{2}(t)=\left.\boldsymbol{P}(s, t)\right|_{s=l} .
$$

According to the third Newton's law,

$$
\begin{array}{ll}
\boldsymbol{F}\left(A_{1}, A_{3}\right)=\left.\boldsymbol{F}(s, t)\right|_{s=0}, & \boldsymbol{M}\left(A_{1}, A_{3}\right)=\left.\boldsymbol{M}(s, t)\right|_{s=0}, \\
\boldsymbol{F}\left(A_{2}, A_{3}\right)=-\left.\boldsymbol{F}(s, t)\right|_{s=l}, & \boldsymbol{M}\left(A_{2}, A_{3}\right)=-\left.\boldsymbol{M}(s, t)\right|_{s=l} .
\end{array}
$$

Eqs. (24)-(26) describe boundary conditions for partial differential Eqs. (15), (16), (19), (23).

Thus, our mathematical diatomic molecule model described by a partial differential equations is constructed. Mathematically the model represents a boundary value problem in space coordinate. Such problem has an infinite spectrum of eigenfrequencies. Below the problem is considered in a simplified form, allowing, however, to describe the known experimental facts.

\section{Description of infrared molecular spectra using continuum model}

In this section, we consider continuum model of a diatomic molecule, Eqs. (15), (16), (19), (23), (24)-(26). Small free oscillations near the equilibrium position are investigated. The stiffness tensors $\boldsymbol{A}, \boldsymbol{B}, \boldsymbol{C}$ of a medium are taken in the forms:

$$
\boldsymbol{A}=c \boldsymbol{\tau} \boldsymbol{\tau}+G(\boldsymbol{E}-\boldsymbol{\tau} \boldsymbol{\tau}), \quad \boldsymbol{B}=0, \quad \boldsymbol{C}=c_{\tau} \boldsymbol{\tau} \boldsymbol{\tau}+D(\boldsymbol{E}-\boldsymbol{\tau} \boldsymbol{\tau}),
$$

where $\tau$, as before, is the unit vector directed along the axis of a molecule; $\boldsymbol{E}$ is the unit tensor; $c, G, c_{\tau}$, and $D$ are respectively the longitudinal, transversal, torsional, and bending stiffness of the continuum, which connects the atoms in the molecule. The inertia tensors of the atoms and linear densities of inertia tensors of the medium are following:

$$
\begin{aligned}
& m_{1} \boldsymbol{\Theta}_{1}^{(1)}=0, \quad m_{1} \boldsymbol{\Theta}_{2}^{(1)}=J_{1} \boldsymbol{E}, \quad m_{2} \boldsymbol{\Theta}_{1}^{(2)}=0, \quad m_{2} \boldsymbol{\Theta}_{2}^{(2)}=J_{2} \boldsymbol{E}, \\
& \rho \boldsymbol{\Theta}_{1}^{(3)}=0, \quad \rho \boldsymbol{\Theta}_{2}^{(3)}=\theta_{\tau} \boldsymbol{\tau} \boldsymbol{\tau}+\theta(\boldsymbol{E}-\boldsymbol{\tau} \boldsymbol{\tau}) .
\end{aligned}
$$

Here the atoms are assumed to have spherical symmetry, the medium has transversal isotropy.

Using assumptions (27), (28), it can be shown that in linear approximation of the system of Eqs. (15), (16), (19), (23)(26) may be combined into four independent systems. Below, we examine, which of these systems can describe the infrared spectra. 
The first system describes oscillations of a molecule along its axis, namely:

$$
u^{\prime \prime}(s, t)-\frac{\rho}{c} \ddot{u}(s, t)=0,\left.\quad m_{1} \ddot{u}\right|_{s=0}=\left.c u^{\prime}\right|_{s=0},\left.\quad m_{2} \ddot{u}\right|_{s=l}=-\left.c u^{\prime}\right|_{s=l},
$$

where $u(s, t)$ is the displacement of particles in a medium along the axis of a molecule. If the mass of the medium is far less than the mass of atoms, $\rho l \ll m_{1}, \rho l \ll m_{2}$, then the translational inertia properties of the medium can be neglected. Using designations $\left.u\right|_{s=0}=u_{1},\left.u\right|_{s=l}=u_{2}$, the system (29) can be reduced to

$$
\left(u_{2}-u_{1}\right) \cdot+\frac{C}{\tilde{m}}\left(u_{2}-u_{1}\right)=0, \quad C=\frac{c}{l}, \quad \tilde{m}=\frac{m_{1} m_{2}}{m_{1}+m_{2}} .
$$

Eq. (30) is the equation of a harmonic oscillator. As a rule, it describes the strongly pronounced resonant peak in the near infrared spectral area. There are no reasons to refuse this model. Moreover, it coincides well with the experiments on investigation of isotopes. It is known, that isotopes possess identical chemical properties. In the above mentioned model, the interatomic bond stiffness $C$ stands for the chemical properties. Hence, the stiffness $C$ should be identical for all isotopes. The internuclear bond stiffnesses evaluated from experimentally measured frequencies and masses of isotopes shows that chemical properties of diatomic molecule isotopes are really identical. The presence of strips at double and triple frequency in the vibrational spectrum, is explained as a rule by the difference of the molecule as such from a simple harmonic oscillator. On our opinion, in scientific literature, there are no publications on the models describing the mentioned resonant peaks. Probably, solution of the problem can be obtained by means of a continuum model (29). It is needed to examine the case of motion of a molecule with another (not longitudinal) degree of freedoms.

The second and third systems describe bending oscillations of a molecule. The equation for bending oscillations has the fourth order with respect to space coordinates. A corresponding spectroscopic problem cannot have the solution in the form of equidistant spectrum. Therefore, bending oscillations of a molecule cannot be associated with the rotational spectrum. However, accounting of "bending" degrees of freedom allows to describe the second and third frequency for the vibrational spectrum [10]. Unfortunately, detailed discussion of this question is beyond the framework of our work.

The fourth system describes torsional oscillations of a molecule about its axis:

$$
\varphi^{\prime \prime}-\frac{\theta_{\tau}}{c_{\tau}} \ddot{\varphi}=0,\left.\quad J_{1} \ddot{\varphi}\right|_{s=0}=\left.c_{\tau} \varphi^{\prime}\right|_{s=0},\left.\quad J_{2} \ddot{\varphi}\right|_{s=l}=-\left.c_{\tau} \varphi^{\prime}\right|_{s=l},
$$

where $\varphi$ is the angle of rotation of particles about the molecular axis. As it follows from system (31), the torsional oscillations of a molecule are described by a wave equation. The wave equation, under a certain boundary conditions, can give an equidistant spectrum. Let us seek a solution for Eqs. (31) in the form of:

$$
\varphi(s, t)=\Phi(s) \sin (2 \pi \nu t+\alpha) .
$$

Substituting (32) in Eqs. (31) gives the frequency equation, namely,

$$
\left(1-I_{1} I_{2} z^{2} \nu^{2}\right) \sin (z \nu)+\left(I_{1}+I_{2}\right) z \nu \cos (z \nu)=0,
$$

where parameters $I_{1}, I_{2}, z$ have the form

$$
I_{1}=\frac{J_{1}}{\theta_{\tau} l}, \quad I_{2}=\frac{J_{2}}{\theta_{\tau} l}, \quad z=2 \pi l \sqrt{\frac{\theta_{\tau}}{c_{\tau}}} .
$$

It is readily seen that if the inertia moments of atoms are far less than the inertia moments of the medium connecting the atoms, i.e. $I_{1} \ll 1, I_{1} \ll 1$, then the solution of Eqs. (33) will describe an almost equidistant spectrum. Thus, the set of Eqs. (31) describing torsional oscillations of the molecule about its axis, can adequately determine the rotational spectrum in the far infrared spectral area.

\section{Description of a rotational molecular spectrum by means of our continuum model: numerical analysis and comparison with experiments}

Now, we discuss a technique for determination of the model parameters. An elastic medium simulates atomic valence bond in a molecule; it can be considered as a mechanical analogue of an electron cloud bonding nuclei of the atoms. Then, the mass of a medium is equal to the mass of one, two or three electrons, depending on the valency of a bond. The linear density of the moment of inertia in medium $\theta_{\tau}$ can be calculated by means of the following formula: $\theta_{\tau}=2 \pi \int_{0}^{r_{*}} \rho(r) r^{3} d r$, where 
$r_{*}$ is the radius of a electron cloud. It is not possible to define $r_{*}$ and $\rho(r)$ reliably, and, therefore, parameter $\theta_{\tau}$ should be determined from the eigenfrequencies of molecule oscillations. The torsional stiffness of medium $c_{\tau}$ is, also, an unknown parameter of the model and it should be found from the indication of eigenfrequencies.

It is possible to use various physical models for determination of inertia moments of nuclei $J_{1}, J_{2}$. At the first sight, the following model seems to be the most reasonable, where a nucleus is a homogeneous sphere and its radius is proportional to the relative nuclear mass, while density is identical for all elements. In this case, the relation of dimensionless parameters is $I_{1} / I_{2}=\left(m_{1} / m_{2}\right)^{5 / 2}$. However, another model based on the assumption, that nuclei of atoms are spheres of identical radius with an identical law of the mass distribution, gives the best correspondence with experiments. In this case, the ratio of inertia moments of nuclei is equal to the ratio of their masses: $I_{1} / I_{2}=m_{1} / m_{2}$.

Thus, from three parameters of the model, $I_{1}, I_{2}$, $z$, only the ratio $I_{1} / I_{2}$ can be considered as a known one. Obviously, this ratio will depend on the choice of a model for nuclei. In principle, parameters $I_{1}, I_{2}, z$ can be determined by means of experimental values of three eigenfrequencies. However, as quantities $I_{1}, I_{2}$ are small (of the order of $10^{-5}$ ) from the point of view of computations, it is preferable to set the ratio $I_{1} / I_{2}$ a priori. Then, two parameters remained are determined using two eigenfrequencies, whereas by means of the third parameter correctness of the above approximation of ratio $I_{1} / I_{2}$ can be appreciated.

Our technique of determination of parameter $z$ is the following. Three experimentally obtained values of frequencies $\nu_{1}$, $\nu_{2}, \nu_{3}$, we substitute in Eq. (33) and we obtain three equations for parameters $I_{1}, I_{2}, z$. Then, by simple transformations the three equations can be reduced to one transcendental equation for parameter $z$. This equation has an infinite number of solutions, and further it is necessary to choose one of them. So, Eq. (33) can be written in the form:

$$
\operatorname{tg}(z \nu)=\frac{\left(I_{1}+I_{2}\right) z \nu}{I_{1} I_{2} z^{2} \nu^{2}-1}
$$

Note, that in the case with higher $\nu$, the following approximation is valid

$$
\operatorname{tg}(z \nu) \approx 0 \quad \Rightarrow \quad z \nu_{n} \approx \pi n
$$

Thus,

$$
z \approx \frac{\pi}{\nu_{n}-\nu_{n-1}}
$$

Now, among the set of solutions of the transcendental equation it is necessary to choose the value of $z$, which is the closest to (37). It should be noticed that values of $z$ calculated by means of various sets of three of eigenfrequencies appear to be very close (differences are observed in the third significant digit).

To determine parameters $I_{1}, I_{2}$, it is necessary to substitute one of the experimentally known eigenfrequencies, by before found parameter $z$ and relations $I_{2}=\frac{m_{2}}{m_{1}} I_{1}$ in Eq. (33), where the ratio of atomic masses is a known quantity. This gives a quadratic equation for parameter $I_{1}$, where the choice of one of two solutions is obvious. Values of parameters $I_{1}, I_{2}$ are essentially dependent on the choice of frequency, which is used in calculation. However, the values of these parameters, apparently, are so small (of order $10^{-4}-10^{-6}$ ), that an error in their determination does not, practically, influence on values of eigenfrequencies.

For verification of experimental determination of the parameters $I_{1}, I_{2}$, when using the frequencies of the rotational spectrum, it is useful to calculate the parameters by means of a theoretical approach. Let us assume, that the mass of a nucleus is uniformly distributed on the surface of a sphere of radius $R=10^{-15} \mathrm{~m}$. Then, inertia moments of nuclei can be calculated using by formula:

$$
J_{i}^{*}=\frac{2}{3} A_{i} m_{H} R^{2}
$$

where $A_{i}$ is the relative nuclear mass and $m_{H}=1.66 \cdot 10^{-24} \mathrm{~g}$ is the mass of a hydrogen atom. Now, we assume, that the mass of an electron cloud, bonding atoms in the molecule, is uniformly distributed on the surface of a cylinder of radius $r_{*}$ over the length of equilibrium internuclear distance $l$. For determination of quantity $r_{*}$ we accept two assumptions: 1) the density of the electron cloud with the valence bond in a diatomic molecule including $N$ electrons, is $N$ times higher than the density of an electron cloud in an atom of hydrogen; 2) in an atom of hydrogen the mass of an electron is uniformly distributed over the surface of a sphere with radius equals to the radius of atom $r_{H}=3 \cdot 10^{-11} \mathrm{~m}$. Then, $r_{*}=2 r_{H} / l$, and the moment of inertia of the electron cloud cross-section is calculated by the formula:

$$
\theta_{\tau}^{*}=\frac{4 N m_{e} r_{H}^{4}}{l^{3}}
$$


Table 1 Wave numbers for CO-molecule

\begin{tabular}{llll}
\hline $\mathrm{N}$ & $\begin{array}{l}\lambda \cdot 10^{-2}\left[\mathrm{~m}^{-1}\right] \\
\text { (exp. value) }\end{array}$ & $\begin{array}{l}\lambda \cdot 10^{-2}\left[\mathrm{~m}^{-1}\right] \\
\text { (theor. value) }\end{array}$ & rel. err. [\%] \\
\hline 1 & 3.845 & 3.845 & 0 \\
2 & 7.690 & 7.690 & 0 \\
3 & 11.534 & 11.535 & 0.009 \\
4 & 15.379 & 15.381 & 0.013 \\
5 & 19.222 & 19.225 & 0.017 \\
6 & 23.065 & 23.070 & 0.022 \\
7 & 26.907 & 26.915 & 0.030 \\
8 & 30.748 & 30.760 & 0.039 \\
9 & 34.588 & 34.605 & 0.049 \\
10 & 38.426 & 38.450 & 0.062 \\
11 & 42.263 & 42.295 & 0.076 \\
12 & 46.098 & 46.140 & 0.091 \\
13 & 49.932 & 49.985 & 0.106 \\
14 & 53.763 & 53.830 & 0.125 \\
15 & 57.593 & 57.675 & 0.142 \\
16 & 61.420 & 61.520 & 0.163 \\
\hline
\end{tabular}

where $m_{e}=9.10 \cdot 10^{-28} \mathrm{~g}$ is the mass of an electron. According to formulas (34), (38), (39), the dimensionless parameter $I_{i}^{*}=\frac{J_{i}^{*}}{\theta_{\tau}^{*} l}$ is calculated as

$$
I_{i}^{*}=\frac{A_{i} l^{2}}{N} \cdot \frac{m_{H} R^{2}}{6 m_{e} r_{H}^{4}}
$$

By substitution of known values of the mass and radius of an hydrogen atom and the electronic mass and radius of the nucleus, into formula (40), we get

$$
I_{i}^{*}=3.75 \cdot 10^{14} \frac{A_{i} l^{2}}{N} .
$$

The results of calculation of wave numbers $\lambda=c \nu$ (where $c$ is the velocity of light in vacuum) for torsional oscillations of diatomic molecules and comparison of the obtained wave numbers with experimental values of the wave numbers of rotational spectra for the molecules [3] are given below.

CO-molecule. Parameters of the molecule are: $A_{C}=12, A_{O}=16, l=1.128 \cdot 10^{-10} \mathrm{~m}, N=2$. The values of dimensionless parameters, $I_{C}^{*}, I_{O}^{*}$, calculated by formula (41):

$$
I_{C}^{*}=2.86 \cdot 10^{-5}, \quad I_{O}^{*}=3.82 \cdot 10^{-5} .
$$

The values of the dimensionless parameters of our model calculated by means of frequencies of the rotational spectrum:

$$
c z=81.70, \quad I_{C}=3.11 \cdot 10^{-5}, \quad I_{O}=4.14 \cdot 10^{-5} .
$$

Here, parameters $I_{C}, I_{O}$ are determined by the first frequency. Experimental and theoretical values of wave numbers $\lambda=c \nu$ for CO-molecule, and, as well as, relative errors in wave numbers found theoretically are listed in Table 1.

HCl-molecule Parameters of the molecule are: $A_{H}=1, A_{C l}=35, l=1.275 \cdot 10^{-10} \mathrm{~m}, N=1$. The values of dimensionless parameters, $I_{C}^{*}, I_{O}^{*}$, calculated by formula (41):

$$
I_{H}^{*}=6.10 \cdot 10^{-6}, \quad I_{C l}^{*}=2.13 \cdot 10^{-4} .
$$

The values of the dimensionless parameters of our model calculated by means of frequencies of the rotational spectrum:

$$
c z=15.10, \quad I_{H}=7.01 \cdot 10^{-6}, \quad I_{C l}=2.45 \cdot 10^{-4} .
$$


Table 2 Wave numbers for HCl-molecule

\begin{tabular}{llll}
\hline $\mathrm{N}$ & $\begin{array}{l}\lambda \cdot 10^{-2}\left[\mathrm{~m}^{-1}\right] \\
\text { (exp. value) }\end{array}$ & $\begin{array}{l}\lambda \cdot 10^{-2}\left[\mathrm{~m}^{-1}\right] \\
\text { (theor. value) }\end{array}$ & rel. err. [\%] \\
\hline 1 & 20.8 & 20.80 & 0 \\
2 & 41.6 & 41.60 & 0 \\
3 & 62.5 & 62.40 & 0.16 \\
4 & 83.1 & 83.20 & 0.12 \\
5 & 103.7 & 104.00 & 0.29 \\
6 & 124.30 & 124.80 & 0.40 \\
7 & 145.03 & 145.60 & 0.40 \\
8 & 165.51 & 166.40 & 0.54 \\
9 & 185.86 & 187.20 & 0.72 \\
10 & 206.38 & 208.00 & 0.78 \\
11 & 226.50 & 228.80 & 1.02 \\
\hline
\end{tabular}

Table 3 Wave numbers for HF-molecule

\begin{tabular}{llll}
\hline $\mathrm{N}$ & $\begin{array}{l}\lambda \cdot 10^{-2}\left[\mathrm{~m}^{-1}\right] \\
\text { (exp. value) }\end{array}$ & $\begin{array}{l}\lambda \cdot 10^{-2}\left[\mathrm{~m}^{-1}\right] \\
\text { (theor. value) }\end{array}$ & rel. err. [\%] \\
\hline 1 & 41.08 & 41.08 & 0 \\
2 & 82.19 & 82.15 & 0.05 \\
3 & 123.15 & 123.23 & 0.06 \\
4 & 164.00 & 164.30 & 0.18 \\
5 & 204.62 & 205.38 & 0.37 \\
6 & 244.93 & 246.45 & 0.62 \\
7 & 285.01 & 287.53 & 0.88 \\
8 & 324.65 & 328.60 & 1.22 \\
9 & 363.93 & 369.68 & 1.58 \\
10 & 402.82 & 410.75 & 1.97 \\
11 & 441.13 & 451.83 & 2.43 \\
\hline
\end{tabular}

Here, the values of parameters $I_{C}, I_{O}$ are listed, which are calculated using one of the first two frequencies (the values of parameters do not depend on the choice of frequency). Experimental and theoretical values of wave numbers, $\lambda=c \nu$, for $\mathrm{HCl}$-molecule, and relative errors, as well, in wave numbers found theoretically are listed in Table 2.

HF-molecule Parameters of the molecule are: $A_{H}=1, A_{F}=19, l=0.917 \cdot 10^{-10}, N=1$. The values of the dimensionless parameters, $I_{C}^{*}, I_{O}^{*}$, calculated by formula (41):

$$
I_{H}^{*}=3.15 \cdot 10^{-6}, \quad I_{F}^{*}=5.99 \cdot 10^{-5}
$$

The values of the dimensionless parameters of our model calculated by means of frequencies of the rotational spectrum:

$$
c z=7.648, \quad I_{H}=2.82 \cdot 10^{-6}, \quad I_{F}=5.36 \cdot 10^{-5} .
$$

Here, the average values for parameters $I_{C}, I_{F}$ are obtained using the first three frequencies. Experimental and theoretical values of wave numbers $\lambda=c \nu$ for HF-molecule, and, also, relative errors in the theoretically determined wave numbers are listed in Table 3.

As it can be seen in the tables, there is a good agreement of theoretical values with experimental data on the eigenfrequencies. The values of the dimensionless inertia parameters $I$ and $I^{*}$, obtained by different models are even in a more close agreement with each other, than it might be expected in view of infinite small deviation of the spectrum from an equidistant one. 


\section{Qualitative description of thin structure of resonant peak in near infrared spectral area}

Experimental investigations show that a resonant peak in the near infrared spectral area consists of a set of close located resonant peaks. It is possible to explain this fact by a mutual influence of translational and rotational motions. Small oscillations of a diatomic molecule near its equilibrium state were considered above. In this case longitudinal and torsional oscillations of the molecule are described by an independent set of equations. However, in the case, where the rotation of the molecule as a rigid body is taken into account, there the situation will be different. Here we show it. For simplification of mathematical statement of the problem the following three assumptions are used:

1) Bending oscillations of the molecule are neglected. In other words, transversal and bending stiffness are considered to be infinite, i.e. $G \rightarrow \infty$ and $D \rightarrow \infty$.

2) The mass of a medium is small in comparison to the masses of atoms, i.e. $\rho l \ll m_{1}, \rho l \ll m_{2}$. Here we may neglect the inertia of the medium with respect to translational motions.

3) There are no external influences on a molecule. In this case, the center of masses of the molecule moves at constant velocity, while the angular momentum of the molecule calculated with respect to its center of masses to be conserved, i.e. $\boldsymbol{K}_{2}^{C}=\boldsymbol{K}=$ const.

4) At an initial instant the direction of an angular momentum vector is not close to the direction of the molecule axis. In this case, the desired effect of mutual influence of translational and torsional oscillations can be observed.

Now we introduce an inertial frame of reference moving together with the center of molecular mass. Below all equations of motion are written in terms of the frame of reference. The tensor of rotation for particles of medium $\boldsymbol{P}(s, t)$ and tensors of rotation for atoms $\boldsymbol{P}_{1}(t)$ and $\boldsymbol{P}_{2}(t)$ can be represented as [6,7]:

$$
\boldsymbol{P}(s, t)=\boldsymbol{Q}(t) \cdot \boldsymbol{P}_{\tau}(s, t), \quad \boldsymbol{P}_{1}(t)=\left.\boldsymbol{P}(s, t)\right|_{s=0}, \quad \boldsymbol{P}_{2}(t)=\left.\boldsymbol{P}(s, t)\right|_{s=l} .
$$

Here, tensor of rotation $\boldsymbol{P}_{\tau}(s, t)=\boldsymbol{P}(\varphi(s, t) \boldsymbol{\tau})$ describes rotation of particles in the medium about the axis of a molecule, while the angle of rotation $\varphi(s, t)$ defines torsional oscillations and rotation of the molecule about its axis. Tensor of rotation $\boldsymbol{Q}(t)$ can be represented as the composition of two tensors of rotation $[6,7]$

$$
\boldsymbol{Q}(t)=\boldsymbol{Q}_{2}(\psi \boldsymbol{k}) \cdot \boldsymbol{Q}_{1}(\gamma \boldsymbol{m}), \quad \boldsymbol{m}=\boldsymbol{\tau} \times \boldsymbol{k} /|\boldsymbol{\tau} \times \boldsymbol{k}|,
$$

where $k$ is the unit vector of molecular angular momentum. The vector of the angular velocity of particles in medium and vectors of the angular velocity of atoms are calculated as in papers $[8,9]$

$$
\boldsymbol{\omega}(s, t)=\boldsymbol{\omega}_{Q}(t)+\boldsymbol{Q}(t) \cdot \dot{\varphi}(s, t) \boldsymbol{\tau}, \quad \boldsymbol{\omega}_{1}(t)=\left.\boldsymbol{\omega}(s, t)\right|_{s=0}, \quad \boldsymbol{\omega}_{2}(t)=\left.\boldsymbol{\omega}(s, t)\right|_{s=l},
$$

where $\boldsymbol{\omega}_{Q}(t)$ is the angular velocity vector corresponding to the tensor of rotation $\boldsymbol{Q}(t)$. In addition to the quantities defined above, the so-called right vector of angular velocity $\Omega_{Q}(t)=\boldsymbol{Q}^{T}(t) \cdot \boldsymbol{\omega}_{Q}(t)$ is used. Vectors of position of atoms in the frame of reference moving together with the center of molecular mass are following:

$$
\boldsymbol{R}_{1}(t)=\boldsymbol{Q}(t) \cdot\left(\left[-l_{1}+u_{1}(t)\right] \boldsymbol{\tau}\right), \quad \boldsymbol{R}_{2}(t)=\boldsymbol{Q}(t) \cdot\left(\left[l_{2}+u_{2}(t)\right] \boldsymbol{\tau}\right), \quad l_{1}=\frac{m_{2} l}{m_{1}+m_{2}}, \quad l_{2}=\frac{m_{1} l}{m_{1}+m_{2}}
$$

Further, we assume that displacements of the atoms $u_{1}(t), u_{1}(t)$ are small in comparison with the internuclear distance $l$, and all the equations are derived in linear approximation.

Dynamics of the molecule is described by Eqs. (15), (16), (19), (23)-(26). In consideration the assumptions formulated above and elimination the unknown constraint forces, we obtain the set of equations including an equation for longitudinal oscillations

$$
\left(u_{2}-u_{1}\right)^{\cdot \cdot}+\frac{C}{\tilde{m}}\left(u_{2}-u_{1}\right)=\left[\left|\boldsymbol{\Omega}_{Q}\right|^{2}-\left(\boldsymbol{\tau} \cdot \boldsymbol{\Omega}_{Q}\right)^{2}\right]\left(u_{2}-u_{1}+l\right),
$$

and equations for torsional oscillations

$$
\varphi^{\prime \prime}-\frac{\theta_{\tau}}{c_{\tau}} \ddot{\varphi}=\frac{\theta_{\tau}}{c_{\tau}}\left(\boldsymbol{\tau} \cdot \boldsymbol{\Omega}_{Q}\right)^{\cdot},\left.\quad\left(\ddot{\varphi}-\frac{c_{\tau}}{J_{1}} \varphi^{\prime}\right)\right|_{s=0}=-\left(\boldsymbol{\tau} \cdot \boldsymbol{\Omega}_{Q}\right)^{\prime},\left.\quad\left(\ddot{\varphi}+\frac{c_{\tau}}{J_{2}} \varphi^{\prime}\right)\right|_{s=l}=-\left(\boldsymbol{\tau} \cdot \boldsymbol{\Omega}_{Q}\right)^{\prime},
$$

along with the law of conservation of the molecular angular momentum

$$
\boldsymbol{R}_{1} \times m_{1} \dot{\boldsymbol{R}}_{1}+\boldsymbol{R}_{2} \times m_{2} \dot{\boldsymbol{R}}_{2}+J_{1} \boldsymbol{\omega}_{1}+J_{2} \boldsymbol{\omega}_{2}+\int_{-l_{1}}^{l_{2}} \boldsymbol{Q} \cdot\left[\theta_{\tau} \boldsymbol{\tau} \boldsymbol{\tau}+\theta(\boldsymbol{E}-\boldsymbol{\tau} \boldsymbol{\tau})\right] \cdot \boldsymbol{Q}^{T} \cdot \boldsymbol{\omega} d s=\boldsymbol{K}
$$


Solution of Eq. (48) gives

$$
\gamma(t) \equiv 0, \quad \dot{\psi}(t)=\frac{|\boldsymbol{K}|}{J}-\frac{2 \tilde{m} l|\boldsymbol{K}|}{J^{2}}\left(u_{2}-u_{1}\right), \quad J=J_{1}+J_{2}+\theta l+\tilde{m} l^{2} .
$$

In terms of (49) the equation for longitudinal molecular oscillations (46) takes the form:

$$
\left(u_{2}-u_{1}\right)^{*}+\left[\frac{C}{\tilde{m}}-\frac{\left(J-4 \tilde{m} l^{2}\right)(\boldsymbol{\tau} \times \boldsymbol{K})^{2}}{J^{3}}\right]\left(u_{2}-u_{1}\right)=\frac{(\boldsymbol{\tau} \times \boldsymbol{K})^{2} l}{J^{2}} .
$$

The set of Eqs. (47), describing torsional oscillations of the molecule in terms of (49), can be written as

$$
\varphi^{\prime \prime}-\frac{\theta_{\tau}}{c_{\tau}} \ddot{\varphi}=-\frac{2 \theta_{\tau} \tilde{m} l \boldsymbol{\tau} \cdot \boldsymbol{K}}{c_{\tau} J^{2}}\left(u_{2}-u_{1}\right)^{\cdot},\left.\quad\left(\ddot{\varphi}-\frac{c_{\tau}}{J_{1}} \varphi^{\prime}\right)\right|_{s=0}=\left.\left(\ddot{\varphi}+\frac{c_{\tau}}{J_{2}} \varphi^{\prime}\right)\right|_{s=l}=\frac{2 \tilde{m} l \boldsymbol{\tau} \cdot \boldsymbol{K}}{J^{2}}\left(u_{2}-u_{1}\right)^{\circ} .
$$

As it can be seen from Eqs. (50), (51), longitudinal oscillations of the molecule influence on its torsional oscillations. At some certain values of the molecular angular momentum, the multiple resonances can be observed at frequencies of translational motions in the system, which are multiple by the frequencies of torsional oscillations. Since the frequencies of torsional oscillations are much lower, the multiple resonances can give the group of closely located resonant peaks. The thin structure of the resonant peak in vicinity of the eigenfrequency of longitudinal oscillations can be explained by means of the multiple resonances.

\section{Conclusions}

In our work an approach for the description of dynamics of diatomic molecules, based on accounting of inertial properties of an interatomic bond is proposed. With the approach the potential of interaction between atoms in the molecule is defined by a set of partial differential equations. It is shown in the work, that our approach allows to describe molecular spectra in the infrared spectral area within the framework of classical mechanics. A good agreement of theoretical results with experimental data measured in far infrared spectral area and qualitative explanation of a thin structure of the resonant peak in the near infrared spectral area is achieved.

Acknowledgements This work was partially supported by RFBR grant 05-01-00094.

\section{References}

[1] G. Herzberg, Molecular spectra and molecular structure. I. Diatomic molecules (Prentice-Hall, New York, 1939), 592 p.

[2] M.V. Volkenstein, Structure and physical properties of diatomic molecules, Izd. AN SSSR. Moscow - Leningrad, 1955. 638 p (in Russian).

[3] A. A. Ravdel and A. M. Ponomareva (eds.) The brief reference book of physical and mathematical quantities "Ivan Fedorov", 2003. 240 pp. (in Russian).

[4] E. A. Ivanova, A. M. Krivtsov, N.F. Morozov, and A.D. Firsova Inclusion of the Moment Interaction in the Calculation of the Flexural Rigidity of Nanostructures, Doklady Physics 48(8), 455-458 (2003).

[5] E.A. Ivanova, A. M. Krivtsov, N.F. Morozov, and A.D. Firsova, Decsription of crystal particle packing considering moment interactions, Mech. Solids 38(4), 101-117i(2003).

[6] P.A. Zhilin, A New Approach to the Analysis of Free Rotations of Rigid Bodies, ZAMM. Z. angew. Math. Mech. 76, 187-204 (1996).

[7] P.A. Zhilin, Rigid body oscillator: a general model and some results, Acta Mechanica 142, 169-193 (2000).

[8] P.A. Zhilin, Theoretical mechanics. Fundamental laws of mechanics. Tutorial book. St. Petersburg State Polytechnical University, 2003. 340 p. (in Russian).

[9] P.A. Zhilin, Advanced problems in mechanics, St. Petersburg, 2006. V. 2. 271 p.

[10] E. A. Ivanova and A. M. Krivtsov, Energy control for polyatomic molecule, in: Control of physical and technical systems (Nauka, St. Petersburg, 2004, in Russian). 